\title{
GEOMETRY OF THE ZEROS OF THE SUMS OF LINEAR FRACTIONS
}

\author{
BY \\ J. L. WALSH( $\left.{ }^{1}\right)$
}

The object of the present paper is to study the zeros of functions of the form

$$
f(z) \equiv \sum_{k=1}^{m} \frac{a_{k}}{z-\alpha_{k}}-\sum_{k=1}^{n} \frac{b_{k}}{z-\beta_{k}}, \quad a_{k}>0, \quad b_{k}>0,
$$

where the $\alpha_{k}$ and $\beta_{k}$ have various geometric configurations as their loci. We investigate also functions of this form where the $a_{k}$ and $b_{k}$ are nonreal.

The appropriateness of this study arises from the facts that (i) Lagrange's interpolation formula for a polynomial with prescribed real values in real points $\alpha_{k}$ and $\beta_{k}$ has a factor of precisely form (1), and a similar remark holds for nonreal values and nonreal points; (ii) Riemann sums for a Cauchy integral are of these same forms, in the respective real and nonreal cases; (iii) the logarithmic derivative of a rational function is of form (1), which enables us to study the location of the critical points. Our main theorems (Theorems 1 and 2) refer respectively to the real and nonreal cases just mentioned, where the locus of the $\alpha_{k}$ and $\beta_{k}$ is a line segment with the $a_{k}$ and $b_{k}$ real, or a circular disk with the $a_{k}$ and $b_{k}$ not necessarily real.

Theorem 2 is a special case of a much more general theorem due to Marden [3], but is proved in detail here particularly because of the applications (i) and (ii), not mentioned by Marden. Namely, the present methods apply also to the case (Theorems 3 and 4) where the locus of the $\alpha_{k}, \beta_{k}$, etc., is a circumference rather than a disk, a case not included in Marden's treatment yet important precisely for the study of a Cauchy or Cauchy-Stieltjes integral.

As is frequently done [2] in the study of zeros of such functions as (1), we interpret the conjugate of $f(z)$ as the force at $z$ due to repelling particles at the $\alpha_{k}$ and attracting particles at the $\beta_{k}$, where each particle repels with a force equal to its mass $a_{k}$ or $-b_{k}$ times the inverse distance; the original problem of finding the zeros of $f(z)$ is equivalent to the problem of finding the positions of equilibrium in this field of force.

Theorem 1. Let the conditions of (1) be satisfied, with $A=\sum a_{k}>B=\sum b_{k}$, and let all $\alpha_{k}$ and $\beta_{k}$ lie on the interval $-1 \leqq z \leqq+1$. Then all nonreal zeros

\footnotetext{
Presented to the Society, September 10, 1962; received by the editors March 22, 1963 and, in revised form, July 3, 1963.

(1) This research was sponsored (in part) by the Air Force Office of Scientific Research.
} 
of $f(z)$ lie in the closed interior of the ellipse

$$
B x^{2}+A y^{2}=\frac{A B}{A-B} .
$$

All real zeros of $f(z)$ lie in the interval

$$
|x| \leqq \frac{A+B}{A-B}
$$

Indeed the sets mentioned constitute the locus of the zeros of $f(z)$ for all $f(z)$ satisfying the hypothesis.

In the field of force already introduced, if a point $z_{0}$ is considered, it is frequently convenient (cf. [2]) to replace $n$ positive (or negative) particles $\alpha_{k}\left(\right.$ or $\beta_{k}$ ) by a single equivalent particle whose mass is the sum of the masses of the original particles and which exerts the same force at $z_{0}$. If the particle $\alpha_{k}$ is inverted in the unit circle whose center is $z_{0}$, the corresponding force at $z_{0}$ is represented by the vector from the inverse of $\alpha_{k}$ to the point $z_{0}$ multiplied by the mass of the particle; the total force at $z_{0}$ due to all the particles $\alpha_{k}$ is represented by the vector (weighted by the total mass) from the center of gravity of the weighted inverses to $z_{0}$; the equivalent particle of the $\alpha_{k}$ is located at the inverse of this center of gravity. We often have occasion to use the fact that if a number of initial points of vectors with common terminal point and various weights are given, their center of gravity lies in their convex hull; this center of gravity is the inital point of the vector resultant, weighted by the sum of weights of the given vectors.

With the hypothesis of Theorem 1 we first choose $\operatorname{Im}\left(z_{0}\right)>0$. For fixed $z_{0}$ the particle $\alpha_{0}$ equivalent to the given $\alpha_{k}$ lies in the circular segment $S\left(z_{0}\right)$ bounded by the interval $-1 \leqq x \leqq 1$ and by an arc of the circle through $-1,+1$, and $z_{0}$ whose endpoints are $z=+1$ and -1 ; the arc lies in the closed half-plane $\operatorname{Im}(z) \leqq 0$. This remark follows from the fact that the inverse in the unit circle whose center is $z_{0}$ of the interval $-1 \leqq x \leqq+1$ is an arc of a circle through $z_{0}$; the convex hull of this arc is a certain segment of a circle whose inverse is $S\left(z_{0}\right)$. Moreover, $S\left(z_{0}\right)$ is the actual locus of the equivalent particle when all possible choices of the $\alpha_{k}$ are considered, not restricted in total number or in respective (positive) masses.

The locus of the particle $\beta_{0}$ equivalent to the $\beta_{k}$ is also $S\left(z_{0}\right)$, and $z_{0}$ is a position of equilibrium if and only if $\alpha_{0}$ and $\beta_{0}$ (in their respective loci) are collinear, with $\left|z_{0}-\alpha_{0}\right| /\left|z_{0}-\beta_{0}\right|=A / B$. If $\alpha_{0}^{\prime}$ and $\beta_{0}^{\prime}$ are any two positions of $\alpha_{0}$ and $\beta_{0}$ collinear with $z_{0}$ and in their proper loci, say with $\beta_{0}^{\prime}$ on the interval $-1 \leqq x \leqq 1$ and $\alpha_{0}^{\prime}$ on the circular arc partially bounding $S\left(z_{0}\right)$, then the ratio $\left|z_{0}-\alpha_{0}\right| /\left|z_{0}-\beta_{0}\right|$ can be increased by rotating the line $z_{0} \alpha_{0} \beta_{0}$ about $z_{0}$, and by sliding $\beta_{0}$ from $\beta_{0}^{\prime}$ along the interval and sliding $\alpha_{0}$ from $\alpha_{0}^{\prime}$ along the circular arc in the sense so as algebraically to decrease the 
ordinate of $\alpha_{0}$; this increase of the ratio is always possible as long as the abscissa of $\alpha_{0}$ is not zero. The maximum of the ratio occurs when $\beta_{0}$ is on the interval, say $\beta_{0}=\beta_{0}^{\prime \prime}$, and when the abscissa of $\alpha_{0}$ is zero, say $\alpha_{0}=\alpha_{0}^{\prime \prime}$. However, the ratio can take on all values and only values between unity and this maximum inclusive, for suitable choices of $\alpha_{0}$ and $\beta_{0}$ in their proper loci and collinear with $z_{0},\left|z_{0}-\alpha_{0}\right| \geqq\left|z_{0}-\beta_{0}\right|$. Thus $z_{0}$ can be a position of equilibrium if and only if we have

$$
\frac{\left|z_{0}-\alpha_{0}^{\prime \prime}\right|}{\left|z_{0}-\beta_{0}^{\prime \prime}\right|} \geqq \frac{A}{B}
$$

If we set $z_{0}=x_{0}+i y_{0}$, and note that the center of the circle an arc of which bounds $S\left(z_{0}\right)$ in part has the ordinate $b=\left(x_{0}^{2}+y_{0}^{2}-1\right) /\left(2 y_{0}\right)$, inequality (4) can be rewritten as

$$
\frac{y_{0}+\left(1+b^{2}\right)^{1 / 2}-b}{y_{0}} \geqq \frac{A}{B},
$$

which is equivalent to

$$
B x_{0}^{2}+A y_{0}^{2} \leqq \frac{A B}{A-B}
$$

this inequality is valid for both $y_{0}>0$ and $y_{0}<0$, so the proof of the first part of Theorem 1 is complete. It may be noted that the foci of the ellipse (2) are $z=+1$ and -1 , and its eccentricity is $[(A-B) / A]^{1 / 2}$; thus the ellipse corresponding to an arbitrary interval as assigned locus of the $\alpha_{k}$ and $\beta_{k}$ is found at once.

If $z_{0}=x_{0}+i y_{0}$ is real and given, say $z_{0}>1$, the maximum of the first member of (4) is $\left(z_{0}+1\right) /\left(z_{0}-1\right)$, and $z_{0}$ can be a zero of $f(x)$ if and only if we have

$$
\frac{z_{0}+1}{z_{0}-1} \geqq \frac{A}{B}
$$

so $z_{0}$ is a zero of some $f(z)$ if and only if we have

$$
z_{0} \leqq \frac{A+B}{A-B}
$$

A similar discussion applies if we have $z_{0}<-1$.

On the other hand, an arbitrary point of $-1 \leqq x \leqq 1$ belongs to the locus; for instance $z=0$ is a zero of the particular function

$$
f(z) \equiv \frac{A}{z-\alpha_{0}}-\frac{B}{z-\beta_{0}}
$$

provided we have merely $A \beta_{0}=B \alpha_{0}$, so $\alpha_{0}$ and $\beta_{0}$ can be chosen positive and as small as desired. Theorem 1 is established. 
Under the hypothesis of Theorem 1 except that now we take $A=B$, the locus of zeros of the totality of the functions $f(z)$ consists nontrivially of the entire plane. Indeed, let $z_{0}$ be a given nonreal point of the plane and let $z_{1}$ be an interior point of the circular segment $S\left(z_{0}\right)$ already defined. We set

$$
f(z) \equiv \frac{1}{z-\left(z_{1}+\delta\right)}+\frac{1}{z-\left(z_{1}-\delta\right)}-\frac{2}{z-\left(z_{1}+\epsilon\right)},
$$

where $|\delta|$ and $|\epsilon|(>0)$ are chosen so small that $z_{1} \pm \delta$ and $z_{1}+\epsilon$ lie within a circle interior to $S\left(z_{0}\right)$. The function $f(z)$ vanishes when $z=z_{1}+\delta^{2} / \epsilon$, and $\epsilon$ and $\delta$ can be so chosen that this number is $z_{0}$. This discussion does not apply if $z_{0}$ is real, but in that case a slight modification of the discussion of (5) does apply, and shows that $z_{0}$ belongs to the locus of zeros of all $f(z)$.

As an application of Theorem 1 we formulate

CoRollary 1. Let $r(z)$ be a rational function of $z$ whose finite zeros and poles lie on the segment $-1 \leqq z \leqq+1$, of respective total orders $A$ and $B$ or $B$ and $A, A>B$. Then all finite nonreal critical points of $r(z)$ lie in the closed interior of the ellipse (2), and all finite real critical points lie in the closed interval (3).

The logarithmic derivative of $r(z)$ is of form (1), where the $\alpha_{k}$ are the zeros of $r(z)$ and the $\beta_{k}$ are the poles, each enumerated a number of times according to its multiplicity, and where all $a_{k}$ and $b_{k}$ are unity. The corollary follows from Theorem 1.

As a second application we have

Corollary 2. Let $f(z)$ be defined by the Stieltjes integral

$$
f(z)=\int_{-1}^{1} \frac{d \sigma(t)}{t-z}, \quad-1 \leqq t \leqq 1,
$$

where the total positive variation of $\sigma(t)$ on $-1 \leqq t \leqq 1$ is $A$ and the total negative variation is $-B, A>B$. Then all finite nonreal zeros of $f(z)$ lie in the closed interior of the ellipse (2), and all real zeros lie in the closed interval (3).

The proof of Corollary 2 follows by considering the partial sums approximating the Stieltjes integral, and by Theorem 1 . If the total negative variation of $\sigma(t)$ is greater than the total positive variation it suffices to consider the zeros of $-f(z)$.

In the proof of theorems such as Theorem 1 on the geometry of zeros of functions, two methods of proof are frequently used: (i) study of the loci of particles equivalent to various categories of particles; (ii) study of the total forces due to various categories of particles. We have just employed method (i), and now proceed to use method (ii) in a different problem.

Theorem 2 (MARDEN). Let the function $f(z)$ be of the form 


$$
f(z) \equiv \sum_{k=1}^{m} \frac{a_{k}}{z-\alpha_{k}}-\sum_{k=1}^{n} \frac{b_{k}}{z-\beta_{k}}+\sum_{k=1}^{p} \frac{i c_{k}}{z-\gamma_{k}}-\sum_{k=1}^{q} \frac{i d_{k}}{z-\delta_{k}},
$$

where all the $a_{k}, b_{k}, c_{k}$, and $d_{k}$ are non-negative. We set $A=\sum a_{k}, B=\sum b_{k}$, $C=\sum c_{k}, D=\sum d_{k}$, and suppose $(A-B)^{2}+(C-D)^{2} \neq 0$. If $\Gamma:|z| \leqq 1$ is the simultaneous locus of the points $\alpha_{k}, \beta_{k}, \gamma_{k}$, and $\delta_{k}$, for all $a_{k}, b_{k}, c_{k}, d_{k}$ satisfying the conditions given, then the locus of the zeros of $f(z)$ is the disk

$$
|z| \leqq \frac{A+B+C+D}{\left[(A-B)^{2}+(C-D)^{2}\right]^{1 / 2}} .
$$

We continue to interpret the conjugate of $f(z)$ as defining a field of force in the $z$-plane. If $z_{0}$ is a zero of $f(z)$, then $\omega z_{0}$ with $|\omega|=1$ is a zero of $f(z)$ with the original $\alpha_{k}, \beta_{k}, \gamma_{k}, \delta_{k}$ replaced by $\omega \alpha_{k}, \omega \beta_{k}, \omega \gamma_{k}, \omega \delta_{k}$, so it is sufficient for us to study $z_{0}=a$, real; we take $a>1$, and then we make a translation of the plane so that $\Gamma$ becomes $\Gamma_{1}:|z+a| \leqq 1$ and $z_{0}$ becomes $z_{1}=0$. The inverse of $\Gamma_{1}$ in the unit circle whose center is $z_{1}$ is

$$
\left|z+\frac{a}{a^{2}-1}\right| \leqq \frac{1}{a^{2}-1},
$$

and the force exerted at $z_{1}$ due to all the particles $\alpha_{k}$ is represented by a vector with initial point $z_{1}$ and terminal point in the disk

$$
C_{1}:\left|z-\frac{a A}{a^{2}-1}\right| \leqq \frac{A}{a^{2}-1} ;
$$

in fact $C_{1}$ is the locus of the terminal points of such vectors for all possible choices of the $a_{k}$ and $\alpha_{k}$, with $A$ fixed; compare [2, p. 13]. The "disk" $C_{1}$ is represented by the formula given even if $A=0$.

Likewise, the locus of the terminal points of the vectors with initial points in $z_{1}$ and representing the force at $z_{1}$ for all possible choices of the $b_{k}$ and $\beta_{k}$ with $B$ fixed is the disk

$$
C_{2}:\left|z+\frac{a B}{a^{2}-1}\right| \leqq \frac{B}{a^{2}-1} .
$$

The locus of the terminal points of the vectors with initial points in $z_{1}$ representing the total force at $z_{1}$ due to the particles at the $\alpha_{k}$ and $\beta_{k}$ is the disk which is the "sum" of $C_{1}$ and $C_{2}$ :

$$
\left|z-\frac{a(A-B)}{a^{2}-1}\right| \leqq \frac{A+B}{a^{2}-1},
$$

in the sense that if $C_{1}$ and $C_{2}$ are the loci of $z_{1}$ and $z_{2}$ then (7) is the locus of $z_{1}+z_{2}$.

By a similar method, and with the note that the conjugate of $f(z)$ defines 
the forces, it follows that the locus of the terminal points of the vectors with initial points in $z_{1}$ representing the total force at $z_{1}$ due to the particles at the $\gamma_{k}$ and $\delta_{k}$ is the disk

$$
\left|z+\frac{i a(C-D)}{a^{2}-1}\right| \leqq \frac{C+D}{a^{2}-1} .
$$

For the two sets of forces we have vectors with initial points in $z_{1}(=0)$ and terminal points whose loci are the respective disks (7) and (8). The total resultant force is represented by a vector whose initial point is $z_{1}$ and the locus of whose terminal points lies in the disk

$$
\left|z-\frac{a(A-B)-i a(C-D)}{a^{2}-1}\right| \leqq \frac{A+B+C+D}{a^{2}-1} .
$$

A necessary and sufficient condition that $z_{1}$ be a possible position of equilibrium is that the total force may be zero, or that $z_{1}(=0)$ should lie in the disk (9), namely

$$
\left|\frac{a(A-B)-i a(C-D)}{a^{2}-1}\right| \leqq \frac{A+B+C+D}{a^{2}-1}, \quad a=\left|z_{0}\right|>1,
$$

which is essentially (6). The second member of (6) is greater than unity unless three of the four numbers $A, B, C, D$ are zero.

If $z_{0}$ is a zero of $f(z)$ for a particular choice of the $\alpha_{k}$ etc., and if $0<\rho<1$, the point $\rho z_{0}$ is a zero of $f(z)$ with the $a_{k}, b_{k}, c_{k}, d_{k}$ unchanged and the $\alpha_{k}$, $\beta_{k}, \gamma_{k}, \delta_{k}$ multiplied by $\rho$. Moreover $z_{0}=0$ is a zero of $f(z)$ with suitably chosen $\alpha_{k}, \beta_{k}, \gamma_{k}, \delta_{k}$ small in modulus, and this completes the proof of Theorem 2 .

CoRollary 1. If $f(z)$ in Theorem 2 is of the form

$$
f(z) \equiv \sum_{k=1}^{m} \frac{a_{k}}{z-\alpha_{k}}+\sum_{k=1}^{n} \frac{i c_{k}}{z-\gamma_{k}}, \quad A+C \neq 0,
$$

the locus of its zeros is the disk

$$
|z| \leqq \frac{A+C}{\left(A^{2}+C^{2}\right)^{1 / 2}} .
$$

Corollary 2. If $f(z)$ in Theorem 2 is of the form

$$
f(z) \equiv \sum_{k=1}^{m} \frac{a_{k}}{z-\alpha_{k}}-\sum_{k=1}^{n} \frac{b_{k}}{z-\beta_{k}}, \quad A>B,
$$

the locus of its zeros is the disk

$$
|z| \leqq \frac{A+B}{A-B} .
$$


If $r(z)$ is a rational function not identically constant, and if the exact degrees of its numerator and denominator are $A$ and $B$, its logarithmic derivative is of form (10); the conclusion of Corollary 2 is essentially that all zeros of the derivative lie in the disk (11), a result [1] proved by the present author in 1918.

If $f(z)$ is multiplied by $\omega$ with $|\omega|=1$, the zeros of the new function $f_{1}(z)$ $(\equiv \omega f(z))$ are unchanged, yet the second member of (6) is not unchanged by such an arbitrary transformation; indeed the denominator in the second member of (6) is precisely $|A-B+i C-i D|$, which is invariant, but the numerator is not invariant. This seeming paradox is resolved if we consider for instance the special case $B=C=D=0$. The original theorem refers to the zeros of $\sum a_{k}\left(z-\alpha_{k}\right)^{-1}, a_{k}>0$ whereas if we set $\omega=\cos \theta+i \sin \theta$, $0<\theta<\pi / 2$; the new function $f_{1}(z)$ is to be written $\sum a_{k}(\cos \theta+i \sin \theta)$ $\cdot\left(z-\alpha_{k}\right)^{-1}, \sum a_{k}=A$, which is quite different from the function

$$
\sum a_{k}^{\prime}\left(z-\alpha_{k}\right)^{-1}+\sum i c_{k}^{\prime}\left(z-\beta_{k}\right)^{-1}
$$

for all $a_{k}^{\prime}, c_{k}^{\prime}$ having prescribed sums $\sum a_{k}^{\prime}=A \cos \theta, \sum c_{k}^{\prime}=A \sin \theta$.

As a consequence of the facts just discussed, we formulate the following

REMARK. In the application of Theorem 2 we may replace $f(z)$ by $\omega f(z)$, where $\omega$ is a constant of modulus unity; this change may modify the second member of (6). In particular if $f(z)$ can be written so as to contain one or more terms of the form

$$
\frac{\lambda_{k}}{z-\zeta_{k}}
$$

where $\arg \lambda_{k}$ is independent of $k$, then as far as those terms are concerned it is favorable to choose $\arg \omega=-\arg \lambda_{k}$.

Corollary 2 to Theorem 1 has an analogue here, concerning the integral

$$
\phi(z) \equiv \int_{\gamma} \frac{d \alpha(t)}{t-z} .
$$

If $\gamma$ lies in the closed interior of the unit circle, if $\alpha(t)=\alpha_{1}(t)+i \alpha_{2}(t)$ where $\alpha_{1}(t)$ and $\alpha_{2}(t)$ are real, and if $A$ and $-B$, and $C$ and $-D$, are the respective total positive and negative variations of $\alpha_{1}(t)$ and $\alpha_{2}(t)$ on $\gamma$, and if $(A-B)^{2}+(C-D)^{2} \neq 0$, then all zeros of the approximating sums of $\phi(z)$ (which approach $\phi(z)$ ) lie in the closed interior of a variable disk that approaches (6), so by Hurwitz's theorem all zeros of $\phi(z)$ lie in the closed interior of (6).

The remarks just made concerning $\phi(z)$ suggest the study of the hypothesis of Theorem 2 except that now the $\alpha_{k}$ and $\beta_{k}$ are required to lie on the unit circumference $\gamma$. If the locus of positive particles $\alpha_{k}$ is $\gamma$, and if $z_{0}$ lies exterior to $\gamma$, the locus of the equivalent particle is the closed interior of $\gamma$, as becomes obvious at once by inversion in the unit circle whose center 
is $z_{0}$. If the locus of these $\alpha_{k}$ is $\gamma$ and $z_{0}$ lies interior to $\gamma$, the locus of the equivalent particle is the closed exterior of $\gamma$ including the point at infinity. If $z_{0}$ lies interior to $\gamma$, we may consider the equivalent particles for each category of particles to lie at infinity, whence $f\left(z_{0}\right)=0$. We have

Theorem 3. Let the hypothesis of Theorem 2 be modified so that all particles $\alpha_{k}, \beta_{k}, \gamma_{k}, \delta_{k}$ lie on $\gamma:|z|=1$. As far as concerns points $z$ not on $\gamma$, the locus of the zeros $z$ of $f(z)$ is the disk (6).

This proof of Theorem 3 involves essentially applying the method of proof of Theorem 2, but not applying Theorem 2 itself.

To study the points $z$ on $\gamma$, we consider (as in the proof of Theorem 2) the actual forces at $z_{0}$ due to the various categories of particles, and the locus of the terminal points of the vectors representing these forces, when the initial points lie in $z_{0}$. We omit the assumption $(A-B)^{2}+(C-D)^{2} \neq 0$. As before, let us choose $z_{0}$ positive and then translate the plane, so that $\gamma$ becomes $|z+1|=1$ and $z_{0}$ becomes $z_{1}=0$. The inverse of $\gamma$ in the unit circle whose center is $z_{1}$ is the line (better, the finite points of the line) $x=-1 / 2$, and the locus of the terminal points of all vectors each corresponding to a set of particles $\alpha_{k}$ is the line $x=A / 2$ unless $A=0$. The locus of the terminal points of all vectors each corresponding to a set of particles $\beta_{k}$ is the line $x=-B / 2$ unless $B=0$, and for the composition of a pair of these vectors we have as locus the line $x=(A-B) / 2$; however, it is to be noted that all vectors are null vectors if we have $A=B=0$. The loci for the vectors corresponding to the $\gamma_{k}$ and $\delta_{k}$ are respectively the lines $y$ $=-C / 2$ and $y=D / 2$ unless $C=0$ or $D=0$; for the composition of a pair of these vectors we have as locus of the terminal points the line $y$ $=-(C-D) / 2$, except if $C=D=0$. The locus for the negatives of these last mentioned vectors having their inital points in $z_{1}$ is $y=(C-D) / 2$, which always intersects the line $x=(A-B) / 2$; the total sum of all vectors is null for a suitable configuration depending on given $A, B, C, D$, with the exceptions noted.

Theorem 4. With the hypothesis of Theorem 3, the locus of the zeros of $f(z)$ contains the entire circumference $\gamma$ provided we have $A+B \neq 0$ and $C+D$ $\neq 0$. The locus contains the entire circumference also if $A=B, C+D=0$, or if $A+B=0, C=D$. The locus contains no point of $\gamma$ if $A \neq B, C+D$ $=0$ or if $A+B=0, C \neq D$.

The case $A=B=C=D=0$ is of course trivial.

It is of interest to indicate how Theorems 1 and 2 apply to the study of zeros of restricted infrapolynomials; for these methods, compare [4], [5]. The category of restricted infrapolynomials on a set $E$ as used here includes the category of similarly restricted polynomials of least norm on $E$, where 
norm is in the sense of least weighted pth powers $(p>0)$ or in the sense of (weighted) Tchebycheff.

Theorem 5. Let the two disjoint point sets $\alpha_{1}, \alpha_{2}, \cdots, \alpha_{m}$ and $\beta_{1}, \beta_{2}, \cdots, \beta_{n}$ consist of the distinct points indicated and lie on $-1 \leqq z \leqq 1$. Let the real polynomial $P(z) \equiv N z^{m+n-1}+\cdots$ have the coefficient $N$ prescribed, and also the (real) values $P\left(\alpha_{j}\right)$, and be a thus restricted infrapolynomial (i.e., have no restricted underpolynomial) on $E:\left\{\beta_{j}\right\}$. Set $\sum_{1}^{m}\left[P\left(\alpha_{j}\right) / \omega^{\prime}\left(\alpha_{j}\right)\right]=N_{0}$, where $\omega(z)$ $\equiv \prod_{1}^{m}\left(z-\alpha_{j}\right) \cdot \prod_{1}^{n}\left(z-\beta_{j}\right)$. Let $A$ and $-B$ be the sum of the positive and negative numbers respectively among $P\left(\alpha_{j}\right) / \omega^{\prime}\left(\alpha_{j}\right), N-N_{0}$; we suppose $A>B$. Then all nonreal zeros of $P(z)$ lie in the closed interior of the ellipse (2), and all real zeros lie in the interval (3).

The polynomial $P(z)$ can be expressed by the Lagrange formula

$$
P(z) \equiv \omega(z) \sum_{1}^{m} \frac{P\left(\alpha_{j}\right)}{\omega^{\prime}\left(\alpha_{j}\right)\left(z-\alpha_{j}\right)}+\omega(z) \sum_{1}^{n} \frac{P\left(\beta_{j}\right)}{\omega^{\prime}\left(\beta_{j}\right)\left(z-\beta_{j}\right)} ;
$$

here the coefficients $P\left(\alpha_{j}\right)$ are prescribed, and the coefficients $P\left(\beta_{j}\right)$ are not prescribed, but are subject to the condition

$$
\sum_{1}^{n} P\left(\beta_{j}\right) / \omega^{\prime}\left(\beta_{j}\right)=N-N_{0} .
$$

It is then clear that for $P(z)$ thus restricted to be an infrapolynomial on $E$ the condition

$$
\operatorname{sg}\left[P\left(\beta_{j}\right) / \omega^{\prime}\left(\beta_{j}\right)\right]=\operatorname{sg}\left(N-N_{0}\right), \quad j=1,2, \cdots, n,
$$

is necessary and sufficient. Indeed, if (15) is satisfied, there exists no restricted underpolynomial $Q(z)$ of $P(z)$ on $E$, for there exists no set of values $Q\left(\beta_{j}\right)$ with

$$
\sum_{1}^{n} Q\left(\beta_{j}\right) / \omega^{\prime}\left(\beta_{j}\right)=N-N_{0}
$$

such that

$$
\left|Q\left(\beta_{j}\right)\right|<\left|P\left(\beta_{j}\right)\right| \text { if } P\left(\beta_{j}\right) \neq 0
$$

and

$$
Q\left(\beta_{j}\right)=0 \quad \text { if } P\left(\beta_{j}\right)=0 .
$$

Conversely, if $P(z)$ is a restricted infrapolynomial and if we have both (14) and $\sum_{1}^{n}\left|P\left(\beta_{j}\right) / \omega^{\prime}\left(\beta_{j}\right)\right|>\left|N-N_{0}\right|$, then we can set

$$
\frac{Q\left(\beta_{j}\right)}{\omega^{\prime}\left(\beta_{j}\right)}=\frac{\left|P\left(\beta_{j}\right) / \omega^{\prime}\left(\beta_{j}\right)\right| \cdot\left|N-N_{0}\right|}{\sum_{1}^{n}\left|P\left(\beta_{j}\right) / \omega^{\prime}\left(\beta_{j}\right)\right|}
$$


whence (16), (17), and (18) are valid and $Q(z)$ is an underpolynomial of $P(z)$ on $E$.

By virtue of (13) with (14) and (15), Theorem 5 now follows from Theorem 1. If a given polynomial $P(z)$ satisfies all the requirements of Theorem 5 except that now $A<B$, we need merely reverse the signs of the $P\left(\alpha_{j}\right)$ and of $N$ to apply Theorem 5 as stated. But we draw no conclusion if $A=B$, namely if $N=0$.

A similar application of Theorem 2, still by use of equations (13), (14), and (15), yields

TheOREM 6. Let the two disjoint point sets $\alpha_{1}, \alpha_{2}, \cdots, \alpha_{m}$ and $\beta_{1}, \beta_{2}, \cdots, \beta_{n}$ consist of the distinct points indicated, and lie in the disk $\Gamma:|z| \leqq 1$. Let the polynomial $P(z) \equiv N z^{m+n-1}+\cdots$ have the coefficient $N$ prescribed, and also the values $P\left(\alpha_{j}\right)$, and be a thus restricted infrapolynomial on $E:\left\{\beta_{j}\right\}$. Let $N_{0}$ and $\omega(z)$ be as defined in Theorem 5 . Let $A, B, C, D$ be respectively the sum of the positive numbers among $\operatorname{Re}[S], \operatorname{Re}[-S], \operatorname{Re}[-i S], \operatorname{Re}[i S]$, where $S$ is the set $\left\{P\left(\alpha_{j}\right) / \omega^{\prime}\left(\alpha_{j}\right), N-N_{0}\right\}$, and where we suppose $(A-B)^{2}+(C-D)^{2}$ $\neq 0$. Then all zeros of $P(z)$ lie in the disk (6).

In connection with Theorem 6, the remark following the proof of Theorem 2 is significant.

\section{REFERENCES}

1. J. L. Walsh, On the location of the roots of the jacobian of two binary forms, and of the derivative of a rational function, Trans. Amer. Math. Soc. 19 (1918), 291-298.

2. The location of critical points, Amer. Math. Soc. Colloq. Publ. Vol. 34, Amer. Math. Soc., Providence, R. I., 1950.

3. M. Marden, On the zeros of the derivative of a rational function, Bull. Amer. Math. Soc. 42 (1936), 400-405.

4. J. L. Walsh and O. Shisha, The zeros of infrapolynomials with prescribed values at given points, Proc. Amer. Math. Soc. 14 (1963), 839-844.

5. Extremal polynomials and the zeros of the derivative of a rational function, Proc. Amer. Math. Soc. 15 (1964), 753-758.

Harvard University, Cambridge, Massachusetts 